\title{
Technical note: A muscle biopsy technique for stratifying cattle by skeletal muscle metabolic activity
}

\author{
L. Beckett, ${ }^{1}$ R. Rosemond, ${ }^{1}$ B. Renquist, ${ }^{2}$ and R. R. White ${ }^{1 *}$ \\ ${ }^{1}$ Department of Animal Science, Virginia Tech, Blacksburg 24061 \\ ${ }^{2}$ Department of Animal and Comparative Biomedical Science, The University of Arizona, Tucson 85721
}

\section{ABSTRACT}

Tissue biopsy metabolic activity, assessed using the oxidation-reduction indicator resazurin, may serve as a proxy to assess energy expenditure associated with maintenance in nongrowing animals or growth rate in growing animals. Herein, we evaluate the repeatability, practicality, and sensitivity of a resazurin-based assay for ranking bovine skeletal muscle biopsies based on metabolic activity. Six yearling Holstein heifers (body weight $=330 \pm 11.3 \mathrm{~kg}$ ) were fed 4 dietary treatments consisting of high or low rumen-degradable starch and fiber arranged factorially in a partially replicated Latin square design. Periods were $18 \mathrm{~d}$, consisting of $3 \mathrm{~d}$ for diet transition, $14 \mathrm{~d}$ for diet adaptation, and $1 \mathrm{~d}$ for sample collection. Semitendinosus biopsies were collected into ice-cold Dulbecco's modified Eagle medium (Fisher Scientific, Hampton, NH) from each heifer during each period. Analysis was initiated within an hour of sample collection. To assess tissue metabolic rate, biopsies were transferred to Dulbecco's modified Eagle medium with resazurin and incubated at $37^{\circ} \mathrm{C}$. Fluorescence of each sample was read at time 0 and at 15-min intervals for $2 \mathrm{~h}$. Change in fluorescence was representative of skeletal muscle reducing equivalent production. Fluorescent signal strength increased with time and relative rank of treatments did not change with time; accordingly, future studies may compare fluorescence at a single time point. Change in fluorescence at $120 \mathrm{~min}$ was used for analysis of the fixed effects of fiber, starch, and animal when accounting for a random effect of period. Samples collected when animals were on a high-ruminally degradable starch diet were more metabolically active than samples collected from animals on low-starch diets. Significant differences in metabolic activity among individual animals were also identified. Average relative fluorescence was correlated with dry matter intake, average daily gain, and feed-togain ratio. The relative fluorescence tended to correlate

Received May 25, 2018.

Accepted December 12, 2018.

*Corresponding author: rrwhite@vt.edu with average daily gain $(\mathrm{r}=0.749)$ and feed-to-gain ratio $(\mathrm{r}=-0.783)$; change in fluorescence did not correlate with dry matter intake. Although evaluated on a small sample size, this technique shows promise as a potential means of ranking animals by growth or feed efficiency. Further work on a larger experimental population is needed to confirm the usefulness of this assay as a consistent and reliable predictor of these important phenotypic parameters.

Key words: bovine, skeletal muscle, metabolic activity

\section{Technical Note}

Selecting feed-efficient animals will help dilute maintenance energy requirements and enhance feed efficiency, improving dairy cattle production system sustainability (White, 2016). One way to select animals with improved feed efficiency is to identify those with particularly efficient metabolism. The energy requirements of cattle are related to maintenance, growth, reproduction, and lactation. Maintenance energy expenditure and efficiency of energy use can be measured by either using metabolic chambers that collect heat and gas produced (Ellis et al., 2006) or performing large serial slaughter studies (Lofgreen and Garrett, 1968). However, the practicality of these approaches has declined over time due to the cost of conducting studies using metabolic chambers and challenges justifying terminal use on large numbers of animals. As a result, our knowledge of how to manipulate energy use efficiency is primarily from cattle with older genetics and older feeding strategies (e.g., Lofgreen and Garrett, 1968). Recent work on the genetic bias of feed efficiency suggests considerable between-animal variation in efficiency of energy use (Hardie et al., 2017). In turn, a high-throughput method for assessing energy expenditure may allow for selection to improve efficiency.

A technique recently developed for measuring metabolic activity in embryonic zebrafish (Renquist et al., 2013; Williams and Renquist, 2016) and skeletal muscle biopsies from tilapia (Kentch et al., 2017) may be applied to assess metabolic activity in cattle, which could serve as a phenotyping approach. In brief, this assay 
applies resazurin, an oxidation-reduction indicator, which is highly fluorescent upon becoming irreversibly reduced by $\mathrm{NADH}, \mathrm{FADH}_{2}$, or cytochrome C. This technique has not previously been tested in cattle. We hypothesized that by applying this technique in cattle skeletal muscle biopsies, we could assess the change in metabolic activity induced by changes in the diet or inherent animal variation in feed efficiency and segregate individuals based on metabolic activity. The objectives of our study were to (1) investigate the application and sensitivity of this novel fluorescent assay using muscle tissue biopsies collected from Holstein heifers; (2) evaluate the application of this test to quantify the metabolic response to dietary changes; and (3) apply the test to identify inherent individual animal variation in metabolic activity.

Six ruminally cannulated yearling $(11 \mathrm{mo} \pm 7 \mathrm{~d})$ Holstein heifers $(\mathrm{BW}=330 \pm 11.3 \mathrm{~kg}$ ) were randomly assigned to 4 dietary treatments in a partially replicated Latin square design. The square was partially replicated because we used 6 animals and 4 treatments, meaning that 2 treatments were duplicated in each period and the other 2 treatments were not. Each period change corresponds to a change in treatment diet. Periods were $18 \mathrm{~d}$ in length. The first $3 \mathrm{~d}$ were used to adapt animals between rations, then animals consumed the treatment ration for $14 \mathrm{~d}$, and samples were collected on the final day. Diets were factorially designed with corn silage as a base $(29.4-35.6 \%$ DM) and dif- ferent dietary inclusion combinations of ground barley [high rumen-degradable starch (HS), 14.1-14.8\% DM; low rumen-degradable starch (LS), $0.310-0.670 \% \mathrm{DM}]$ or ground corn (HS, 0.00-0.380\% DM; LS, 10.5-12.3\% DM) and pelleted beet pulp [high rumen-degradable fiber (HF), 3.55-6.05\% DM; low rumen-degradable fiber (LF), $30.2-32.9 \% \mathrm{DM}$ ] or timothy hay (HF, $19.9-25.0 \%$ DM; LF, $0.020-1.43 \% \mathrm{DM}$ ) to generate rations with high and low ruminal-degradable starch and fiber. As described in Table 1, diets also contained soybean meal (HS-HF, 9.3\% DM; HS-LF, 17.1\% DM; LS-HF, 9.95\% DM; LS-LF, $15.3 \%$ DM), blood meal (HS-HF, 3.78\% DM; HS-LF, 0.00\% DM; LS-HF, 4.37\% DM; LS-LF, $0.040 \% \mathrm{DM}$ ), and corn gluten feed (HS-HF, 3.26\% DM; HS-LF, 0.00\% DM; LS-HF, $1.65 \%$ DM; LS-LF, $7.22 \%$ $\mathrm{DM})$ to make them isonitrogenous. These treatment combinations resulted in divergent energy supply to the animals (NRC, 2001), and the calculated metabolic energy for each ration is included in Table 1.

A 10-cm wide area, 5 to $35 \mathrm{~cm}$ ventral to the point of the ischium, was shaved and scrubbed 3 times with betadine and isopropanol. Ten milliliters of lidocaine was administered subcutaneously in 5 to 6 locations radially arrayed $2 \mathrm{~cm}$ external to the biopsy site. The target biopsy site was $20 \mathrm{~cm}$ ventral to the point of the ischium. Muscle tissue samples were collected by making a 1-cm incision through the skin with a \#20 scalpel blade, inserting a 20-gauge biopsy needle (Bard Mission Disposable Core Biopsy Instrument, Bard Biopsy,

Table 1. Ingredients and nutrient composition for each treatment $\operatorname{diet}^{1}$ expressed on a DM basis

\begin{tabular}{lcccc}
\hline Item & HS-LF & HS-HF & LS-LF & LS-HF \\
\hline Ingredient, \% of DM & & & & \\
Timothy hay & 0.020 & 19.9 & 1.43 & 25.0 \\
Beet pulp & 30.2 & 6.05 & 32.9 & 3.55 \\
Ground corn & 0.000 & 0.380 & 12.3 & 10.5 \\
Ground barley & 14.8 & 14.1 & 0.310 & 0.670 \\
Corn silage & 35.6 & 34.4 & 29.4 & 35.2 \\
Corn gluten feed & 0.000 & 3.26 & 7.22 & 1.65 \\
Soybean meal & 17.1 & 9.30 & 15.3 & 9.95 \\
Blood meal & 0.000 & 3.78 & 0.040 & 4.37 \\
Vitamin E premix & & & & \\
Vitamin A-D premix & & & & \\
Nutrient, \% of DM (unless noted) & 73.4 & 64.8 & 74.7 & 63.9 \\
DM, \% & 94.8 & 94.9 & 94.9 & 94.7 \\
OM & 35.0 & 35.0 & 35.0 & 35.0 \\
NDF & 23.4 & 23.0 & 21.1 & 21.5 \\
ADF & 20.0 & 20.0 & 20.0 & 20.0 \\
Starch & 16.0 & 15.9 & 16.0 & 15.9 \\
CP & 2.74 & 2.63 & 2.75 & 2.62 \\
Estimated ME, Mcal/kg & & & 10.9 & 12.3 \\
Animal parameters & 10.6 & 11.6 & 0.76 & 0.60 \\
DMI, kg/d & 0.90 & 0.81 & 14.3 & 20.5 \\
ADG, kg/d & 11.8 & 14.3 & & \\
Feed-to-gain ratio, kg/kg & & & & \\
\hline
\end{tabular}

${ }^{1}$ HS-LF = high-rumen-degradable starch, low-fiber treatment; HS-HF = high-rumen-degradable starch, highfiber treatment; LS-LF = low-rumen-degradable starch, low-fiber treatment; LS-HF = low-rumen-degradable starch, high-fiber treatment. 
Tempe, AZ) to a $4 \mathrm{~cm}$ depth, and depressing the needle collection sheath to obtain a sample. Three biopsies, with the aim of obtaining approximately $30 \mathrm{mg}$ of sample, were collected per animal per period to evaluate how variation in sample collection (sample mass, collection site within the muscle, and other unknown factors) influenced consistency of the results. Samples were not weighed after collection because an analytical balance was not available at the farm. The incision site was sealed using monofilament \#2 suture wire, cleaned with isopropanol, and sprayed with adhesive bandage. The right semitendinosus was sampled in periods 1 and 3 and the left semitendinosus was sampled in periods 2 and 4, allowing muscle to properly regenerate and animals to heal.

Immediately following collection, biopsies were placed in individual wells of a 96-well plate filled with a pretest solution. The pretest solution was formulated as described in Renquist et al. (2013) and contained $30 \mathrm{~mL}$ of Dulbecco's modified Eagle medium (Fisher Scientific, Hampton, NH), 7.5 mg of Fungizone (Fisher Scientific), $0.12 \mathrm{mg}$ of chloramphenicol (Fisher Scientific), and $0.03 \mathrm{mg}$ of ampicillin (Fisher Scientific). After all samples were collected, they were transferred from the pretest solution into individual wells of a 96well plate filled with AlamarBlue Test Assay solution (Thermo Fisher Scientific, Waltham, MA). The test solution was identical to the pretest solution with $1.6 \%$ AlamarBlue added. Solutions were mixed immediately before biopsy collection, filtered using a sterile $0.22-\mu \mathrm{m}$ filter, and warmed to $37^{\circ} \mathrm{C}$ before use.

Directly after sample collection, we transported the live muscle tissue samples to the laboratory in the pretest solution on ice, transferred to the test solution, and analyzed. Analysis was initiated within $1 \mathrm{~h}$ of tissue collection. When samples were transferred to the test solution, the test solution plate was incubated in the plate reader (Spectramax M5; Molecular Devices LLC, San Jose, CA) at $37^{\circ} \mathrm{C}$. Fluorescence was read at time 0 and every $15 \mathrm{~min}$ for $2 \mathrm{~h}$ using excitation and emission wavelengths of 530 and $590 \mathrm{~nm}$, respectively (Renquist et al., 2013; Williams and Renquist, 2016). Soft Max Pro 6.1 (Molecular Devices LLC) was used to quantify resulting emissions, and relative fluorescence (standardized to time 0) was calculated at each time point for each sample.

Triplicate samples were collected within an animalperiod to evaluate the consistency of animal rankings obtained from alike samples. The within-animal-period coefficient of variation for relative fluorescence was approximately $20 \%$, suggesting a need to standardize samples by protein or DNA content, as was done by Renquist et al. (2013), or by mass. Despite the degree of variation among samples collected from the same animal consuming the same diet, a Wilcoxon rank sum test did not find significant differences in the rankings of animals when different individual samples were used $(P>0.05)$, suggesting this variation did not impede opportunities to rank animals by skeletal muscle metabolic activity.

Mean relative fluorescence was calculated for each animal-period at each time point read. Statistical analysis of these mean fluorescence data was conducted in $\mathrm{R}$ version 3.1.0. ( $\mathrm{R}$ Core Team, 2014). Analysis was structured into 2 questions: (1) does the incubation time change the perceived influence of a treatment on relative $\mathrm{NAD}^{+}$reduction rate and (2) are differences between animals and treatments discernable after 2 h. To address the first question, a linear mixed effect model was used to test how time, fiber and starch digestibility, animal, and period influenced standardized fluorescence readings. Fiber, starch, and time were fixed effects, and the 2- and 3-way interactions among these fixed effects were also evaluated. Period and animal were treated as random effects. A significant time by starch or fiber interaction would suggest that treatment differences were not consistent across a sampling period and some ideal fluorescence reading time would need to be determined to make reliable inferences from the data. To address the second question, data from the last time point $(2 \mathrm{~h})$ were analyzed using a linear mixed effect model with fixed effects of fiber, starch, and animal and a random effect of period. Significant starch, fiber, or animal effects would suggest that the assay (using only $2 \mathrm{~h}$ of fluorescence time) was sensitive enough to rank samples based on animal-level factors (genetic potential or diet).

Because the plate can be read at multiple time points, we were able to test the effect of time on withinsample fluorescence (Figure 1). The reduction reaction that causes resazurin to fluoresce is irreversible; as a result, the signal accumulates with time, allowing small short-term differences to amass as time of incubation is extended. The total signal increased linearly with time $(P<0.0001)$. Thus, the tissue biopsies maintained a constant metabolic rate throughout the incubation period. Time did not differentially affect fluorescence based on starch $(P=0.5106)$ or fiber $(P=0.8072)$ in the diet, supporting the conclusion that the rankings of samples were similar if evaluated at any time point within the $2 \mathrm{~h}$.

Skeletal muscle biopsies taken when heifers were on a high-ruminally degradable starch diet had a higher relative fluorescence than biopsies taken when heifers were fed a diet low in ruminally degradable starch $(P=0.023$; Table 2). The link between starch source and skeletal muscle metabolic activity could be caused by a change in energy availability. However, calculated metabolic 


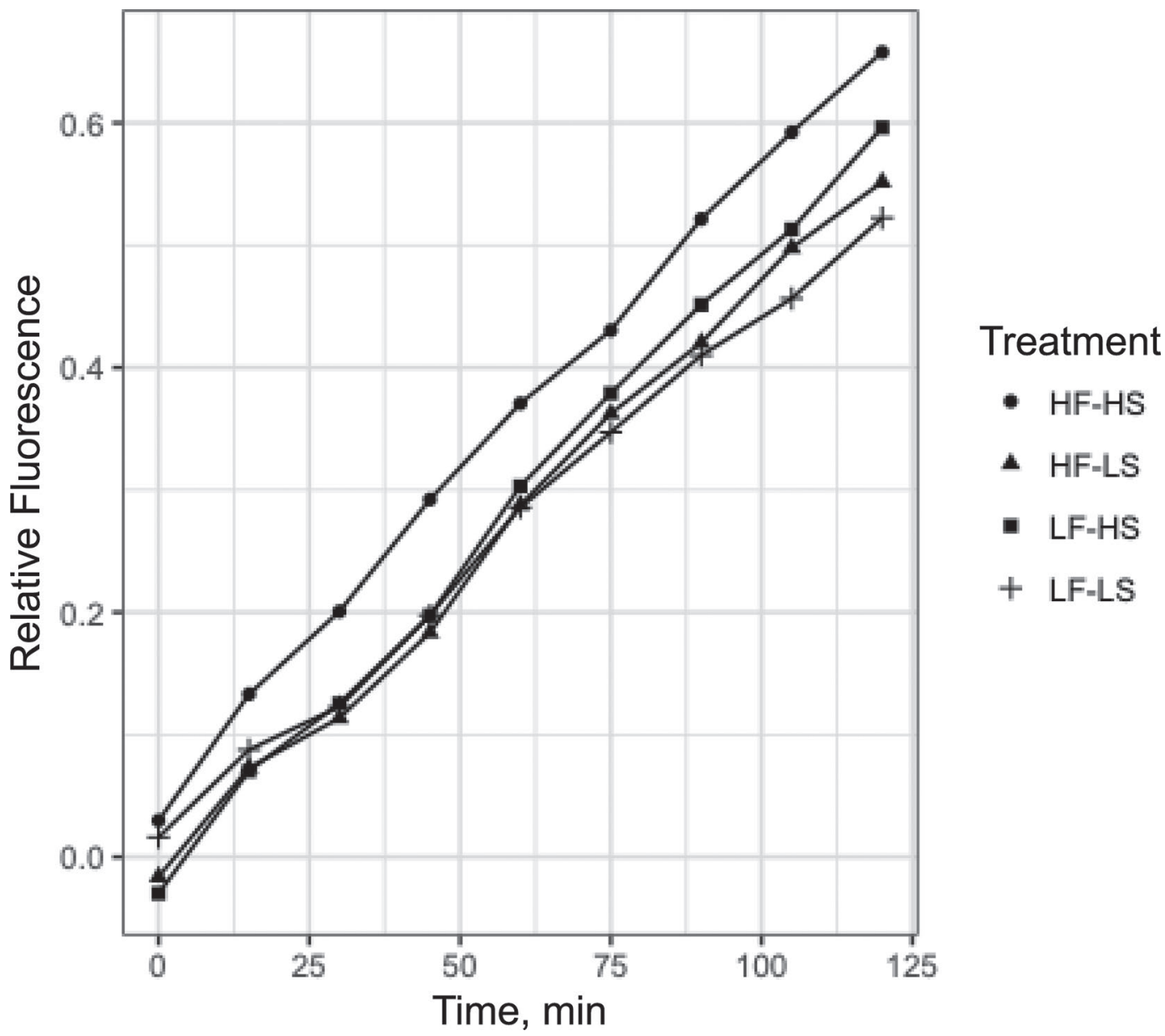

Figure 1. Relative fluorescence, equivalent to energy expenditure, analyzed over a 2-h period for high fiber-high starch (HF-HS), high fiberlow starch (HF-LS), low fiber-high starch (LF-HS), and low fiber-low starch (LF-LS) diets. Values reported are LSM; the raw values for time point 0 were 0 for all treatments, as would be expected from relative fluorescence data.

energy in the diets changed more with rumen-degradable fiber than with rumen-degradable starch (Table 1), making this explanation unlikely. Alternatively, the increase in tissue metabolic activity with increased ruminally degradable starch may be related to a different absorbed VFA profile. Different VFA are used for energy with different efficiencies in the postabsorptive system (Baldwin, 1968). Approximate VFA contribution to muscle is dependent upon individual VFA metabolism. Less than $30 \%$ of acetate (Knowles et al., 1974), 40 to $55 \%$ of propionate (Wiltrout and Satter, 1972), and minimal butyrate are available to the periphery (Bergman and Wolff, 1971). Glucose contribution to skeletal muscle in ruminants is due to gluconeogenesis. Hepatic uptake of propionate, valerate, and isobutyrate allows for increased gluconeogenic substrate (Aschenbach et al., 2010). If the different starch sources contributed to different profiles of absorbed VFA, it is possible that the absorbed VFA profiles contributed to the effect of ruminally degradable starch on skeletal muscle metabolic activity. Independent of the mechanism driving this dietary effect, our results suggest the assay can be applied to understand the metabolic effects of ration changes.

Comparisons among animals show that skeletal muscle metabolic activity varied between individuals $(P=$ 0.003). The effect of diet was consistent among animals and animals ranked similarly across diets (Figure 2). This consistency suggests that inherent differences in skeletal muscle metabolic activity may be distinguishable across conditions, making it a possible way to inexpensively and durably screen animals for energy expenditure associated with growth or, in nongrowing animals, maintenance.

The metabolic activity of a ruminant is a function of body mass and metabolic flux in an inactive, thermoneutral environment (White and Seymour, 2003). These differences in metabolic flux contribute to variability 
Table 2. Relative fluorescence treatment means, SE, and $P$-values for measuring skeletal muscle metabolic activity in bovines fed different rumen-degradable starch and fiber $\operatorname{diets}^{1}$

\begin{tabular}{lccc}
\hline Treatment & $\begin{array}{c}\text { Relative } \\
\text { fluorescence }\end{array}$ & SE & $P$-value \\
\hline HS-LF & 0.567 & 0.038 & \\
HS-HF & 0.687 & 0.038 & \\
LS-LF & 0.537 & 0.038 & \\
LS-HF & 0.537 & 0.038 & \\
Starch & & & 0.023 \\
Fiber & & & 0.226 \\
Starch $\times$ fiber & & 0.120 \\
Animal & & 0.003 \\
\hline
\end{tabular}

$\overline{{ }^{1} \mathrm{HS}-\mathrm{LF}}=$ high-rumen-degradable starch, low-fiber treatment; HS-HF = high-rumen-degradable starch, high-fiber treatment; LS-LF = lowrumen-degradable starch, low-fiber treatment; LS-HF = low-rumendegradable starch, high-fiber treatment.

in feed efficiency. Feed efficiency is also influenced by genetics (e.g., Koch et al., 1963), activity and behavior (e.g., Socha et al., 2007; Shaver, 2010), environment (e.g., Socha et al., 2007), and heifer-rearing practices (e.g., Khan et al., 2011). The average skeletal muscle metabolic activity (expressed as relative fluorescence) of each individual was paired with DMI, ADG, and calculated feed to grain ratio (F:G; Figure 3). Relative fluorescence tended to correlate with ADG ( $\mathrm{r}=$ $0.749 ; P=0.0864)$ and $\mathrm{F}: \mathrm{G}(\mathrm{r}=-0.783 ; P=0.066)$. Change in fluorescence did not correlate with DMI $(P$ $=0.773$; Figure 3 ). Because of concern with potential high-influence points, a leave-out-one analysis was performed to assess how correlations changed when each point was iteratively removed from the data set. Correlations between relative fluorescence and ADG ranged from 0.701 and 0.855 with a mean of 0.778 . The
F:G correlations ranged from -0.540 and -0.875 with a mean of -0.763 . The relationship between DMI and relative fluorescence varied more substantially (range $=0.186-0.542$, mean $=0.187)$. These results suggest that 1 animal may have been the primary reason for limited relationship between DMI and relative fluorescence. When this animal was removed, the strength of the F:G relationship also decreased. However, removing data from that animal did not substantially alter the relationship identified between relative fluorescence and ADG. The inconsistency across the data set suggests the need for additional trials to confirm the relationship between the outputs of this assay and animal productivity.

Skeletal muscle biopsy metabolic activity was indicative of energy expenditure for growth and maintenance in these young, rapidly growing heifers. The tendency for a consistent positive correlation with growth suggests that variation in the metabolic activity assay measurements correlated with variation in animal growth, as would be expected from the measurement. However, the inconsistency of correlations among the relative fluorescence and DMI or F:G highlights a limitation of the small sample size used in this preliminary assay evaluation. Additional screening on a larger sample size is needed to determine whether this assay could be useful in selecting animals with higher rates of gain or greater feed efficiency.

\section{ACKNOWLEDGMENTS}

Research was supported by state and federal funds appropriated to Virginia Tech and by USDA-NIFA

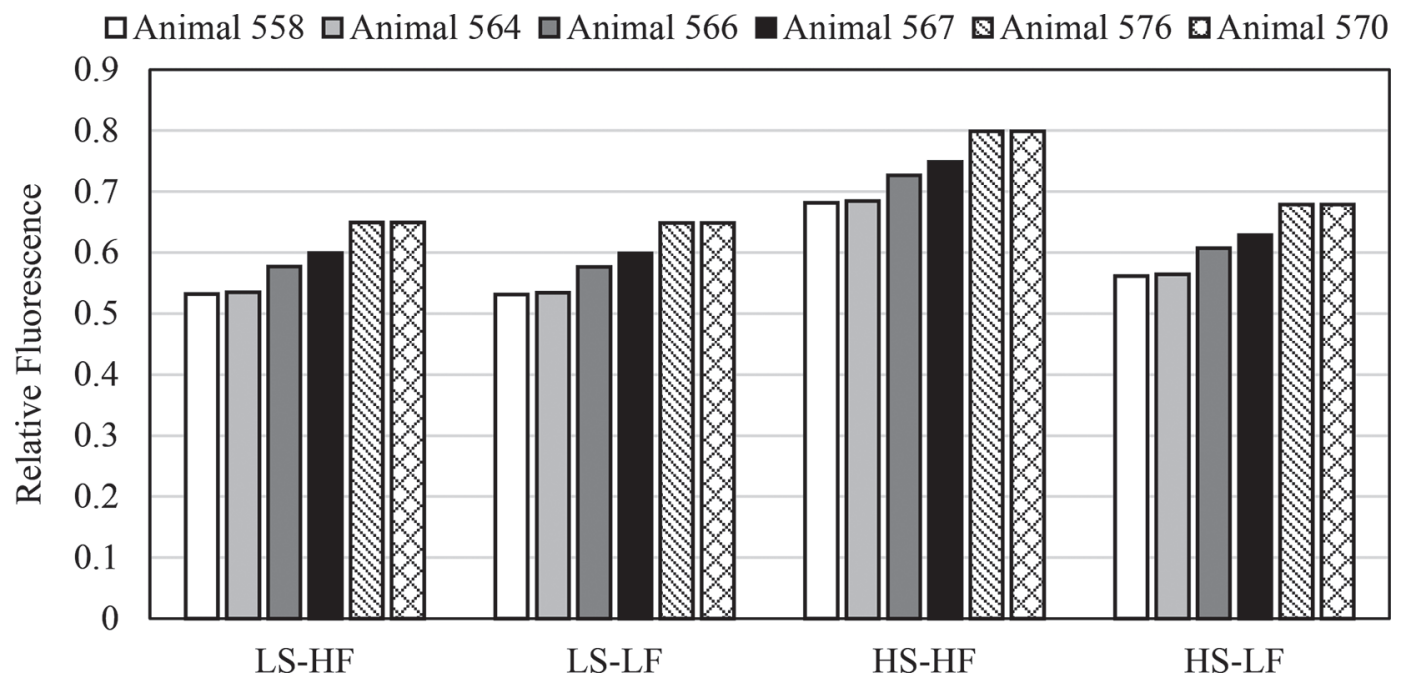

Figure 2. Least squares means relative fluorescence (nm) for each animal, organized by treatment. Treatments included either a high (HS) or low (LS) rumen-degradable starch source and either a high (HF) or low (LF) rumen-degradable fiber source. Animal numbers are arbitrary and reflect the animal identifier assigned by the farm. These identifiers are included in the legend to show consistence of animal rankings across diets. 

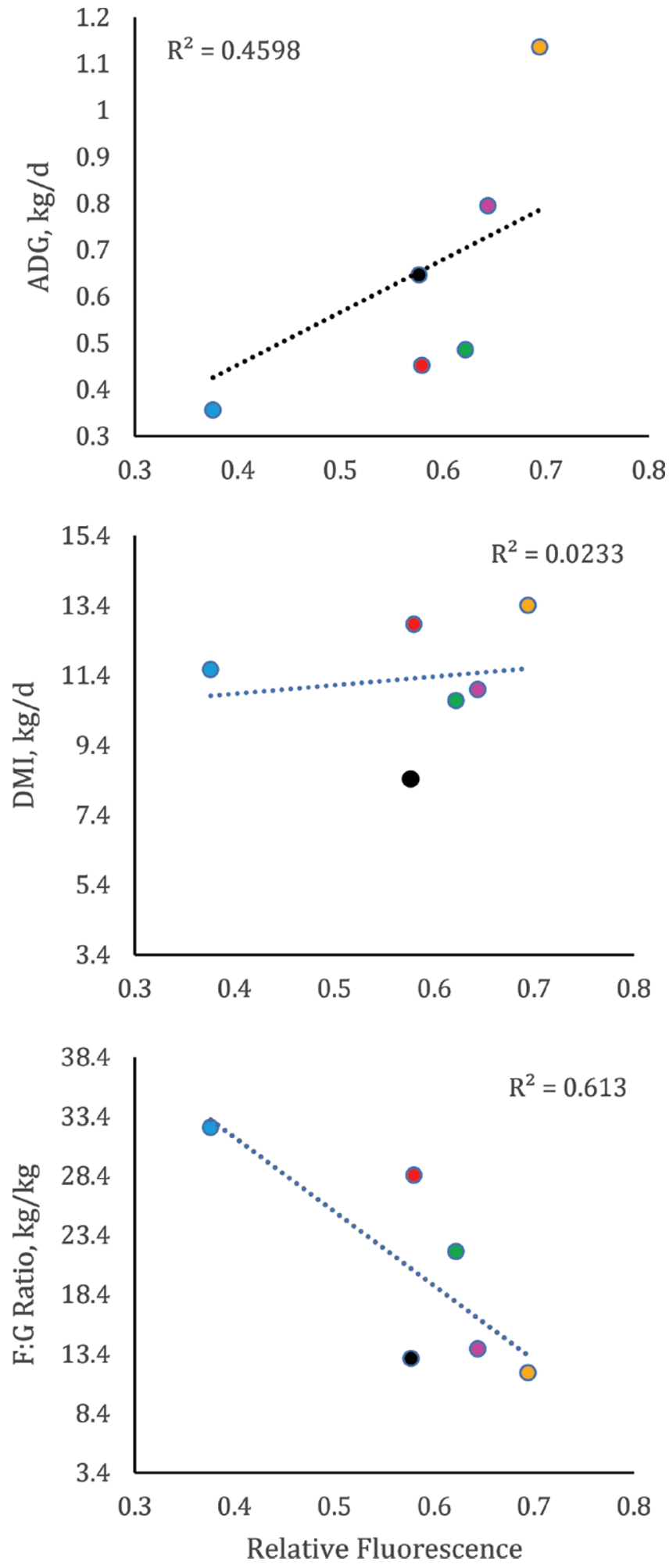

Figure 3. Mean relative fluorescence of each animal mapped against corresponding study mean DMI, ADG, and mean feed-to-gain ratio (F:G). Data points describe individual animal performance correlated with relative fluorescence. Each color corresponds to an individual animal. The dotted line shows a best-fit linear regression of the performance variable on relative fluorescence. The $\mathrm{R}^{2}$ values reflect each regression.
(Washington, DC) project numbers 2015-03656, 201705943 (RRW) and 2015-06367 (BJR).

\section{REFERENCES}

Aschenbach, J. R., N. B. Kristensen, S. S. Donkin, H. M. Hammon, and G. B. Penner. 2010. Gluconeogenesis in dairy cows: The secret of making sweet milk from sour dough. IUBMB Life 62:869-877.

Baldwin, R. L. 1968. Estimation of theoretical calorific relationships as a teaching technique. A review. J. Dairy Sci. 51:104-111.

Bergman, E. N., and J. E. Wolff. 1971. Metabolism of volatile fatty acids by liver and portal-drained viscera in sheep. Am. J. Physiol. 221:586-592.

Ellis, J. L., F. Qiao, and J. P. Cant. 2006. Evaluation of net energy expenditures of dairy cows according to body weight changes over a full lactation. J. Dairy Sci. 89:1546-1557.

Hardie, L. C., M. J. VandeHaar, R. J. Tempelman, K. A. Weigel, L. E. Armentano, G. R. Wiggans, R. F. Veerkamp, Y. de Haas, M. P. Coffey, E. E. Connor, M. D. Hanigan, C. Staples, Z. Wang, J. C. M. Dekkers, and D. M. Spurlock. 2017. The genetic and biological basis of feed efficiency in mid-lactation Holstein dairy cows. J. Dairy Sci. 100:9061-9075.

Kentch, K., C. E. Geisler, and B. J. Renquist. 2017. Measuring metabolic rate of embryonic fish to predict growth rate. Page 359 in Aquaculture America 2017, San Antonio, TX. World Aquaculture Society, Sorrento, LA.

Khan, M. A., D. M. Weary, and M. A. G. von Keyserlingk. 2011. Invited review: Effects of milk ration on solid feed intake, weaning, and performance in dairy heifers. J. Dairy Sci. 94:1071-1081.

Knowles, S. E., I. G. Jarrett, O. H. Filsell, and F. J. Ballard. 1974. Production and utilization of acetate in mammals. Biochem. J. 142:401-411.

Koch, R. M., L. A. Swiger, and D. Chambers. 1963. Efficiency of feed use in beef cattle. J. Anim. Sci. 22:486-494.

Lofgreen, G. P., and W. N. Garrett. 1968. A system for expressing net energy requirements and feed values for growing and finishing beef cattle. J. Anim. Sci. 27:793-806.

NRC. 2001. Nutrient Requirements of Dairy Cattle. 7th ed. Natl. Acad. Sci., Washington, DC.

$\mathrm{R}$ Core Team. 2014. A language and environment for statistical computing. Version 3.1.0. R. Foundation for Statistical Computing, Vienna, Austria.

Renquist, B. J., C. Zhang, S. Y. Williams, and R. D. Cone. 2013. Development of an assay for high-throughput energy expenditure monitoring in the zebrafish. Zebrafish 10:343-352.

Shaver, R. 2010. Factors influencing feed efficiency in dairy cattle. Pages 43-50 in Mid-South Ruminant Nutrition Conference, Arlington, TX. Texas Animal Nutrition Council, Champaign, IL.

Socha, M. T., D. J. Tomlinson, and J. M. DeFrain. 2007. Measuring and improving feed efficiency in lactating dairy cows. Pages 235-246 in Proceedings of the Intermoutain Nutrition Conference, Salt Lake City, UT. Utah Agricultural Experiment Station, Utah State University, Logan, UT.

White, C. R., and R. S. Seymour. 2003. Mammalian basal metabolic rate is proportional to body mass $2 / 3$. Proc. Natl. Acad. Sci. USA 100:4046-4049.

White, R. R. 2016. Increasing energy and protein use efficiency improves opportunities to decrease land use, water use, and greenhouse gas emissions from dairy production. Agric. Syst. 146:20-29.

Williams, S. Y., and B. J. Renquist. 2016. High throughput danio rerio energy expenditure assay. J. Vis. Exp. 27:e53297.

Wiltrout, D. W., and L. D. Satter. 1972. Contribution of propionate to glucose synthesis in the lactating and nonlactating cow. J. Dairy Sci. 55:307-317. 